\title{
Protocol
}

\section{Implementing a low-carbohydrate diet in adults to manage type 1 diabetes mellitus: a standard of care protocol}

\author{
Amanda A. Felizardo ${ }^{1}$, Caroline D. S. B. Paiva ${ }^{2}$, Jordana D. F. L. Brasileiro ${ }^{3}$, \\ Mariana S. D. D. Souza ${ }^{4}$, Rúbia M. Correa ${ }^{3}$, Dayse R. D. Carvalho ${ }^{3}$, Maria V. C. D. Abreu ${ }^{1}$, \\ Laryssa R. D. S. Franckilin ${ }^{1}$, Roberta D. M. V. D. Assis ${ }^{1}$, Francisco D. A. D. M. Júnior ${ }^{5}$, \\ Daniela D. G. Neri ${ }^{3}$, Janaína Koenen ${ }^{3}$, Giselle Foureaux ${ }^{1 *}$
}

\author{
${ }^{1}$ Department of Morphology, Federal University of Minas Gerais, MG, Brazil \\ ${ }^{2}$ University of Sao Paulo, SP, Brazil \\ ${ }^{3}$ The Clinic, Belo Horizonte, MG, Brazil \\ ${ }^{4}$ Gynecology and Obstetrics Service of Dr. Kleber Domingues, RJ, Brazil \\ ${ }^{5}$ Laboratory of exercise physiology, Federal University of Ouro Preto, MG, Brazil
}

Received: 30 August 2021

Revised: 04 November 2021

Accepted: 11 January 2022

\section{*Correspondence: \\ Dr. Giselle Foureaux, \\ E-mail: gifoureaux@icb.ufmg.br}

Copyright: ( $)$ the author(s), publisher and licensee Medip Academy. This is an open-access article distributed under the terms of the Creative Commons Attribution Non-Commercial License, which permits unrestricted non-commercial use, distribution, and reproduction in any medium, provided the original work is properly cited.

\section{ABSTRACT}

Background: Type 1 diabetes mellitus is an autoimmune disease characterized by the destruction of pancreatic beta cells and absolute insulin deficiency. After the discovery of insulin, guidelines recommended $50 \%$ to $60 \%$ of carbohydrate consumption in individuals with diabetes. However, this recommendation is ineffective, since carbohydrate is the macronutrient that causes the greatest increase in blood glucose. The aim of this research is to evaluate the efficacy of a low-carbohydrate diet in adults with type 1 diabetes mellitus.

Methods: The study is a 26 weeks clinical trial. During the first 15 days, participants will receive approximately $130 \mathrm{~g}$ of daily carbohydrates, followed by a phase of 7 days, with the consumption of $90 \mathrm{~g}$ of daily carbohydrates, and finally, the consumption of $50 \mathrm{~g}$ of carbohydrates. The target audience is 20 participants diagnosed with type 1 diabetes mellitus, glycated hemoglobin (HbA1c) >7.0\% and aged between 21 and 50 years, in addition to the parameters defined in the established inclusion and exclusion criteria. The primary outcome is HbA1c. Glycemic variability, frequency of hypoglycemia, total daily insulin and improvement in quality of life will also be evaluated.

Conclusions: Despite the evidence that low carbohydrate is effective for treating type 2 diabetes, there is low evidence for the recommendation in people with type 1 diabetes. In order to improve quality of life of this group and guidelines of clinical practice, and to promote development of health care professionals, further well-designed trials are needed. Trial registration: The trial was registered at https://ensaiosclinicos.gov.br/rg/RBR-107jk4tn.

Keywords: Type 1 diabetes mellitus, Low-carbohydrate diet, Glycemic control

\section{INTRODUCTION}

Type 1 diabetes is an autoimmune disease that usually appears in childhood, but can affect individuals of almost all ages. ${ }^{1}$ The disease is characterized by the destruction of the beta cells of the pancreas, responsible for insulin production, causing chronic hyperglycemia, which, if left untreated, can lead to death., ${ }^{2,3}$ According to the International diabetes federation there are approximately 463 million people with diabetes worldwide. ${ }^{4}$ Diabetes is considered a leading cause of blindness, kidney failure, heart attacks and lower limb amputations. ${ }^{5}$ Data from the Brazilian society of diabetes that Brazil is the fourth country with the largest number of individuals with 
diabetes between 20 and 79 years old. ${ }^{6}$ Approximately 1.6 million Americans have type 1 diabetes, being 187,000 of those children and adolescents. ${ }^{7}$ The rate of new cases each year is 1.5 million Americans. ${ }^{7}$ The economic impact of these complications is exorbitant; in 2019, diabetes accounted for US\$ 760 billion in health expenditures (SBEM, 2019). That representing 10\% of global health expenditures, which include the high utilization of health services, loss of productivity, and long-term care required to treat its chronic complications, such as renal failure, blindness, heart problems and diabetic foot. ${ }^{6}$ The main symptoms of type 1 diabetes are polydipsia, polyphagia, polyuria, weight loss, and fatigue resulting from defective glucose transport from the bloodstream to tissues. ${ }^{8}$ In addition to these symptoms, growth deficit and susceptibility to infections are present in people with type 1 diabetes, with chronic hyperglycemia. ${ }^{9}$ One of the first symptoms that can appear in people with type 1 diabetes is diabetic ketoacidosis, a serious complications of diabetes. ${ }^{10}$ Diabetic ketoacidosis occurs when stored triglycerides are broken down into fatty acids, which causes an increase in blood ketone and leads to ketoacidosis. ${ }^{10,11}$ In developed countries, the mortality rate from Coronary artery disease (CAD) varies from $2 \%$ to $5 \%$, and in developing countries, this ranges from $6 \%$ to $24 \% .^{12}$ Studies show that CAD can be present, at the onset of the disease, in $25 \%$ to $30 \%$ of cases of type 1 diabetes. $^{13}$

Glycemic control and achieving normal glycated hemoglobin $(\mathrm{HbAlc} \leq 7.0 \%$ or $53 \mathrm{mmol} / \mathrm{mol})$ are the strongest predictors of control of diabetes complications, but this goal seems to be far from reality. ${ }^{14,15}$ Studies show that $60 \%$ to $90 \%$ of people with type 1 diabetes have inadequate glycemic control, with $\mathrm{HbA} 1 \mathrm{c}>7.0 \% .{ }^{16-18}$ The biggest challenge in diabetes is to reach $\mathrm{HbA} 1 \mathrm{c}$ levels $<7 \%$, whereas high levels are associated with chronic complications related to hyperglycemia. ${ }^{19}$ Studies show that $84 \%$ of type 1 diabetics in 19 countries HbA1c above $7 \% .^{20}$ For every $1 \%$ of $\mathrm{HbA} 1 \mathrm{c}>7.0 \%$, cardiovascular mortality in people with type 1 diabetes increases by $52 \% .^{22}$ Treatment for people with type 1 diabetes consists of daily applications of insulin doses. ${ }^{22,23}$ However, despite insulin replacement therapy being essential for the survival of people with type 1 diabetes, and delaying complications in the short and long term, insulin therapy still presents challenges. Exogenous insulin replacement, even with the use of an insulin pump, still cannot effectively and accurately replace the kinetics of insulin secretion performed by the pancreatic beta cells. ${ }^{24}$ Furthermore, high amounts of insulin used to achieve glycemic control goals have impacting consequences for long-term health, such as hyperinsulinemia, which is directly associated with obesity, atherosclerosis and metabolic syndrome. ${ }^{22,23}$ Additionally, large amounts of insulin increase the risk of hypoglycemia. ${ }^{25}$ Constant episodes of hypoglycemia affect the quality of life of people with diabetes and result in difficult control of blood glucose..$^{26,27}$ Ultimately, therapies that complement and minimize the use of insulin for the treatment of type 1 diabetes are needed to reduce long-term complications and provide better quality of life for individuals with diabetes.Diabetes awareness and management is still one of the main problems faced worldwide among people with type 1 diabetes, especially in developing countries. ${ }^{28,29}$ Although there are no recommended diet for people with type 1 diabetes, the general guidelines appear not to be effective, according to a range of studies that demonstrate a high prevalence of dyslipidemia, risks for cardiovascular disease, and other complications from diabetes. ${ }^{30,31}$ Nutritional therapy is an adjuvant therapy and is important in the control of diabetes, along with the practice of physical exercise. ${ }^{31}$ Dietary practices among people with diabetes are mainly influenced by cultural backgrounds. ${ }^{32}$ Conventionally, there are data in the literature that show the impact of nutrition on people with diabetes. ${ }^{33}$ Although the few studies available in the literature do not present a consensus on the effects of low-carbohydrate nutritional strategies in people with type 1 diabetes, there is evidence that shows a relationship between the high consumption of carbohydrates to greater glycemic imbalances. ${ }^{34,35}$ Carbohydrate is the main macronutrient that determines insulin dose before meals and postprandial glycemic response, considering that $100 \%$ of carbohydrate is converted to glucose. ${ }^{36}$ The few findings in the literature demonstrate that adherence to a low-carbohydrate diet provided a reduction in body mass index (BMI), less variability in blood glucose measurements, higher concentration of high density lipoprotein (HDL) cholesterol and lower diastolic blood pressure. ${ }^{15}$

According to the American Diabetes Association, a lowcarbohydrate diet is defined by consumption $<130 \mathrm{~g}$ of carbohydrate per day or $26 \%$ of the total energy intake. ${ }^{37}$ A study by, of a retrospective cohort, with 48 people with type 1 diabetes and $\mathrm{HbA} 1 \mathrm{c}<6.1 \%$, in a four-year follow$\mathrm{up}$, in which individuals received an average of $<75 \mathrm{~g}$ of daily carbohydrates, showed that participants who adhered to the diet and did not give up. A total of 13 participants with type 1 diabetes; had a significant improvement in the reduction of hypoglycemia, in addition to a significant reduction in $\mathrm{HbA} 1 \mathrm{c}$, of $7.9 \%$ for $6.0 \% .{ }^{37}$ In a study by Krebs and colleagues, adult DM1 participants were randomized to a standard (no restrictions) diet, where the participants mean carbohydrate intake was $203 \pm 92$ g/day) or to a carbohydrate-restricted diet $(75 \mathrm{~g}$ of carbohydrates/day). ${ }^{39}$ After 12 weeks, the group receiving a carbohydrate-restricted diet showed significant reductions in daily insulin doses, $\mathrm{HbA} 1 \mathrm{c}$, and body weight, in addition to no change in glycemic variability. ${ }^{39}$ Observational studies with 1,020 European outpatients with type I diabetes showed that lower carbohydrate intake was associated with lower levels of HbA1c.40 Demonstrated that the consumption of approximately $70 \mathrm{~g}$ to $90 \mathrm{~g}$ of carbohydrates per day in participants with type 1 diabetes, for 12 months, allowed a significant reduction in the amounts of insulin, hypoglycemia, and A1c. ${ }^{14} \mathrm{~A}$ 2018 systematic review, evaluating the studies in the literature on the low-carbohydrate diet, revealed that none of the studies considered in the review showed any statistically significant harm against the low-carbohydrate 
diet; the effectiveness of the low-carbohydrate diet in the management of type 1 diabetes. ${ }^{14}$ Thus, despite the individuality of the diabetes management, there is a lack of studies to prove the effectiveness of the lowcarbohydrate diet in this context. ${ }^{14}$ It is extremely important to seek treatments that are promising to provide better quality of life for individuals with type 1 diabetes; and to reduce complications arising from inadequate control. From a socioeconomic point of view, it is less costly to invest in diabetes education and prevention than to treat the direct complications (amputations, hemodialysis, and blindness) or indirect complications (diabetes as a complicating factor for any other acute or chronic comorbidity) of the disease.

\section{METHODS}

\section{Type of study}

The longitudinal study is characterized as a clinical trial, carried out in samples from different regions in Brazil, lasting 26 weeks, in participants diagnosed with type 1 diabetes mellitus, aged between 21 and 50 years.

\section{Sample size}

For analysis of the sample calculation, the Gpower software (3.1) was used, considering a test power of $95 \%$ and a significance of 5\%, and was combined; with the basis for the values of variance and mean of glycated hemoglobin, published by Nielsen et al to obtain the number of 20 individuals required for the study. Student's $t$ test; will be used for the initial comparison between the first and last results, which were made after treatment with the low-carbohydrate diet. The analysis of variance (ANOVA) test will used to compare the mean of the sample population and identify there are statistically significant differences, considering $\mathrm{p}<0.05$. Analyses will be performed with the aid of the GraphPad Prism software.

\section{Materials and reagents required to participate in the research protocol}

It included: diet sheets built considering the gender and height of the individuals, standardized on $130 \mathrm{~g}, 90 \mathrm{~g}$ and $50 \mathrm{~g}$ daily carbohydrate diets, including four meals a day: breakfast, lunch, afternoon snack and dinner (provided by the team); basal insulins (except NPH), regular, fast and ultra-fast; glucose measuring strips; lancing device and lancets; syringe for insulin BD ultra-fine $6 \mathrm{~mm}$ graduated $1 / 2$ in $1 / 2$ unit; insulin pens; sugar; gli-instan liquid sugar; and glycemic map (provided by the team).

\section{Equipments needed to participate in the research protocol}

It included: $10 \mathrm{~kg}$ precision digital kitchen scale; scale for body weight; glucose meter (glucometer); and FreeStyle Libre glucose sensor and reader.

\section{Study design}

The recruitment of participants with type 1 diabetes will be carried out through social media networks, more specifically, through the research's Instagram profile, Bdiet (@bdiet.project), created by the team. There will be several posts in stories and in the Instagram feed to advertise the research and recruit participants. Interested parties will undergo a careful assessment by anamnesis through an online questionnaire (https://docs.google.com/forms/d/1mynqcg2oDAzylyBZx kJkoaYKQSzkbkj6R4ZKI_3jYc/edi). In this questionnaire, the participant is instructed to answer several questions.

This anamnesis is evaluated by the team (endocrinologist, nurse, nutritionist and $\mathrm{PhD}$ student) with the goal of being introduced to the participant, learning her general health status, and verifying whether she meets the previously defined inclusion and exclusion criteria. The informed consent form (FICF) will be made available to all participants or guardians to authorize participation in the research. Signatures will be collected by receiving the consent form via email.

\section{Inclusion and exclusion criteria}

Participants will be recruited through social media networks, using the research's Instagram profile, Bdiet. project, and selected by a detailed anamnesis questionnaire, following the inclusion and exclusion criteria, previously determined.

\section{Inclusion criteria}

Patients having a diagnosis of type 1 diabetes mellitus; present from 21 to 50 years of age of both sexes; have $\mathrm{Hb} 1 \mathrm{Ac}>7.0 \%$; agree to follow all parameters considered in the protocol for the treatment of type 1 diabetes with a low-carbohydrate diet; belong to any region of the country; have creatinine clearance $<60$; use a glucometer or sensor (Libre, flash glucose monitoring) for minimum of 7 measurements per day; perform a fundus examination for the diagnosis of diabetic retinopathy; have mild retinopathy treated (to be evaluated by the ophthalmologist and endocrinologist); be willing to consume, during the first 15 days of the protocol, the amount of $130 \mathrm{~g}$ of daily carbohydrates; sign the authorization to participate in the research through the informed consent form (TCLE); and agree to perform all laboratory tests before starting the survey, three months after the start of the $50 \mathrm{~g}$ daily carbohydrate phase, and at the end of the sixth month of the survey were included in the study.

\section{Exclusion criteria}

Patients who have moderate to advanced diabetic retinopathy (to be evaluated by the ophthalmologist and endocrinologist); present reports or suspicion of gastroparesis; have an eating disorder, such as binge 
eating; be an athlete; use an insulin pump; and have a pregnancy diagnosis were excluded.

\section{Diabetes education}

Before starting treatment, the participant will go through a process of "diabetes education" to learn the importance of the disease, with the making them a manager of their selfcontrol, to improve glycemic control and avoid short-term and long-term complications. To achieve this goal, the diabetes manual was created, as well as videos on important topics of diabetes produced by the team members and posted on the research's YouTube page (@bdiet.project), which can be found at this link: https:// www.youtube.com/channel/UCeUVjCTyV0Iv1ZnzbhaI_ uw/.

\section{Biochemical and anthropometric standards}

After confirming that the participant meets the previously established inclusion and exclusion criteria, the endocrinologist issues a request for laboratory tests required to assess the participant's general health. In the first consultation with the endocrinologist, the participant should already have the exams in hand, to expedite the initiation of the treatment. In addition to type 1 diabetes, the participant's general health is assessed and treated by the physician. Table 1 provides the list of laboratory tests requested from the participant. In addition to these tests, fructosamine will be dosed monthly. The same tests will be performed in the participant when she completes 3 months of treatment and at the end of the sixth month of the research protocol. In addition to biochemical tests, the participant will record weekly the weight $(\mathrm{kg})$ until the end of the treatment.

\section{Endocrinology physician, nutritionist and nurse}

The treatment protocol lasts for 26 weeks, during which four appointments with the endocrinologist will take place. The first appointment will be before the start of the treatment protocol, the second within fifteen days after the start, the third at the end of the $13^{\text {th }}$ week, and, the last appointment at the end of the $26^{\text {th }}$ week, that is, at the end of the proposed treatment. During these consultations, glycemic control will be evaluated, as well as any relevant issues that may occur regarding the participant's general health. With the nutritional team, three consultations will be to explain and detail the proposed diet. In addition to these scheduled appointments, the participant will have daily support from the nutritional team. The nurse will also offer consultation, in which the participants will be taught the counting of carbohydrates, proteins, and fats, insulin dose adjustments, among other important parameters. In addition to the consultations, the participant will be monitored daily, 24 hours a day, through WhatsApp groups, which will comprise of the entire healthcare team and the participant. The participant will post photos of all meals.

\section{Phases and duration of treatment}

\section{Phase 0 or control phase}

It is the phase in which the participant will consume daily approximately $130 \mathrm{~g}$ of carbohydrates for 15 days, distributed in 4 daily meals: breakfast, lunch, snack and dinner. The meals will be standardized by the nutritional team, and evaluated according to the participant's individual needs. This phase is considered the control phase. For the control phase, laboratory tests will also be considered for the participant to be performed at time 0 , before starting the treatment.

\section{Phase 1}

After phase 0 or control phase, the participant will consume, approximately, $90 \mathrm{~g}$ of daily carbohydrates for 7 days, distributed in 4 daily meals: breakfast, lunch, snack and dinner. The meals; standardized by the nutritional team, and evaluated according to the participant's individual needs.

\section{Phase 2}

In this phase, the participant diabetes will consume, approximately, $50 \mathrm{~g}$ of carbohydrates daily; until the $26^{\text {th }}$ week of the research protocol, to assess daily blood glucose, glycated hemoglobin (Hb1 Ac), reduction of doses of basal and bolus insulins, meals, hypoglycemia and improvement in quality of life.

\section{Phase 3}

Phase in which the participant is expected to consume, daily, $0 \mathrm{~g}$ of carbohydrates. The participant will only participate in this phase; if necessary. If the participant will only participate in this phase; if necessary. If the participant reaches the glycemic goal consuming $50 \mathrm{~g}$ of carbohydrates daily, and there is no recommendation for a ketogenic diet, participation in this phase will not be required. All these phases are described in Figure 1.

\section{Guidelines for treatment during the six-month research protocol}

\section{Check blood glucose}

Blood glucose should be checked at the "fingertip" with the glucometer, and with blood glucose sensors such as the FreeStyle Libre glucose meter, because correction of hypoglycemia should not always be relied solely on the sensor. The purpose of the two parameters established for measuring glucose is to provide original data with lower chance of errors.

\section{Glycemic map}

The blood glucose map (Figure 2) is an important document in which the participant will record all their 
daily blood glucose levels. To develop this protocol, a glycemic map was created and standardized according to the research needs. Information recorded in the glycemic map include at least 9 daily blood glucose measurements, the physical exercise performed and its duration, and the insulinization used before the practice of physical activity. The instruction of insulinization for the practice of physical activity must be standardized by the endocrinologist and the nurse, considering the participant's individual needs.

\section{Glycemic target and glycemic range}

The glycemic goal is the values that the multidisciplinary team (endocrinologists, nurses, and other healthcare professionals) determines, together with the participant, for different periods of the day. In this protocol, the goal is $100 \mathrm{mg} / \mathrm{dl}$. The standardized glycemic range is from 70 $\mathrm{mg} / \mathrm{dl}$ to $180 \mathrm{mg} / \mathrm{dl}$. That means that the participant must spend most of the time between $70 \mathrm{mg} / \mathrm{dl}$ and $180 \mathrm{mg} / \mathrm{dl}$.

\section{Sensitivity factor}

Sensitivity factor measure how blood glucose lower in $\mathrm{mg} / \mathrm{dl}$, with 1 unit of insulin. In this protocol, the initial sensitivity factor of 50 or 100 was standardized for different situations. For postprandial, hypoglycemia rebounds, post-exercise blood glucose and in the period from 10:00 pm to 6:00 am, sensitivity factor of 100 is consider. In other situations, the sensitivity factor 50 was adopted. Regardless of the proposed sensitivity factor, throughout the study, each participant's individual sensitivity factor will be determined as needed.

\section{Insulin: carbohydrate ratio}

The protocol starts with the insulin to carbohydrate ratio of $1: 15$, that is, 1 unit of insulin for $15 \mathrm{~g}$ of carbohydrates. This ratio only a starting point; the real insulin to carbohydrate ratio will be determined and, refined for each participant throughout the protocol, respecting the individuality of each participant. The same insulin to carbohydrate ratio for the lunch may not be same as the ratio for the afternoon snack, that is, a different insulin to carbohydrate ratio may be needed for each meal. It is worth emphasizing the importance of individuality in this decision.

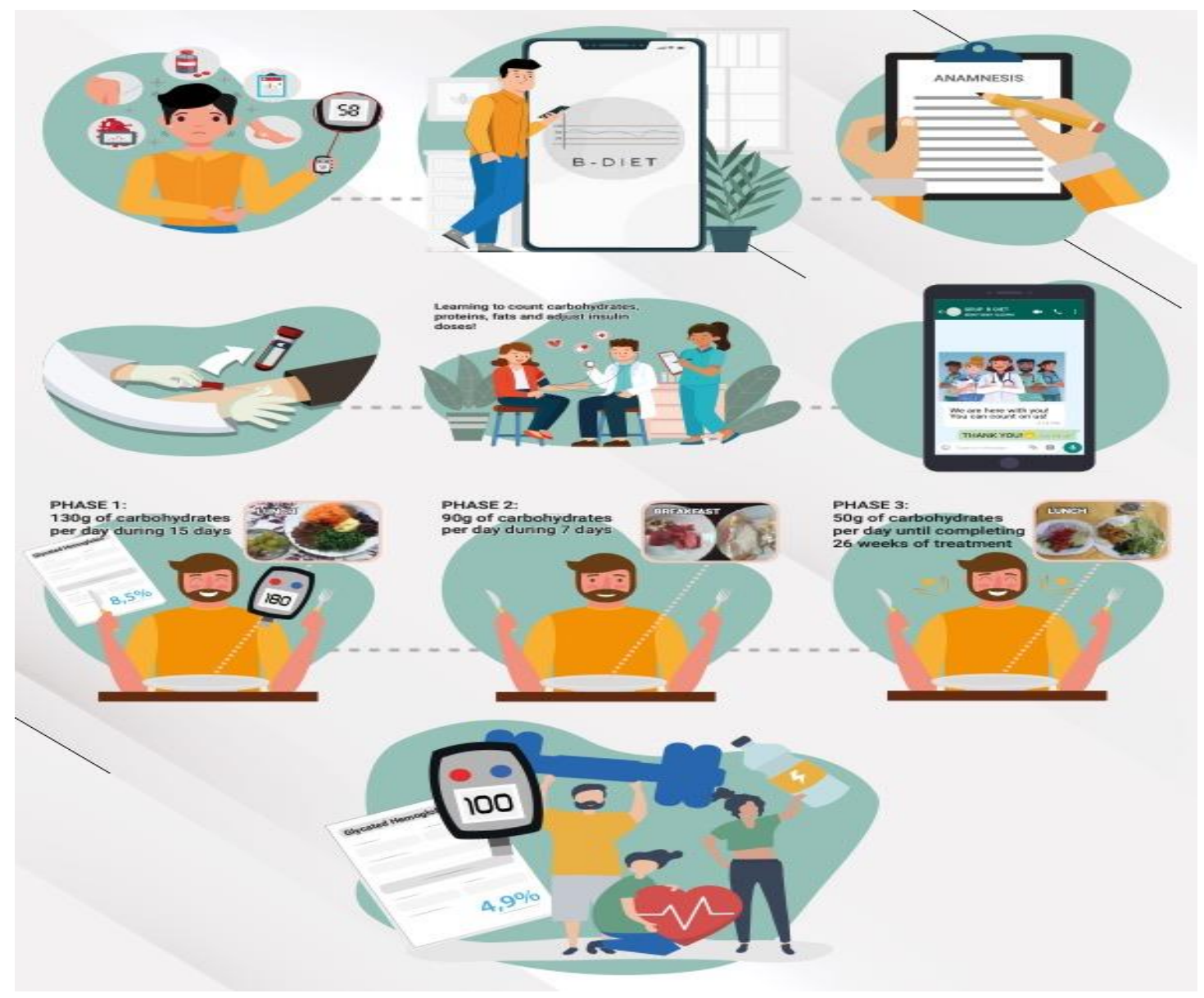

Figure 1: Protocol phases: in phase 1, the patient will consume, approximately, $130 \mathrm{~g}$ of daily carbohydrates for 7 days, distributed in 4 daily meals; in phase 2, the patient will consume, approximately $90 \mathrm{~g}$ of carbohydrates daily; in phase 3, the patient can be submitted to the consumption of $50 \mathrm{~g}$ of carbohydrates daily; and in phase 3 , the patient can be submitted to the consumption of $30 \mathrm{~g}$ of carbohydrates daily, if necessary. 


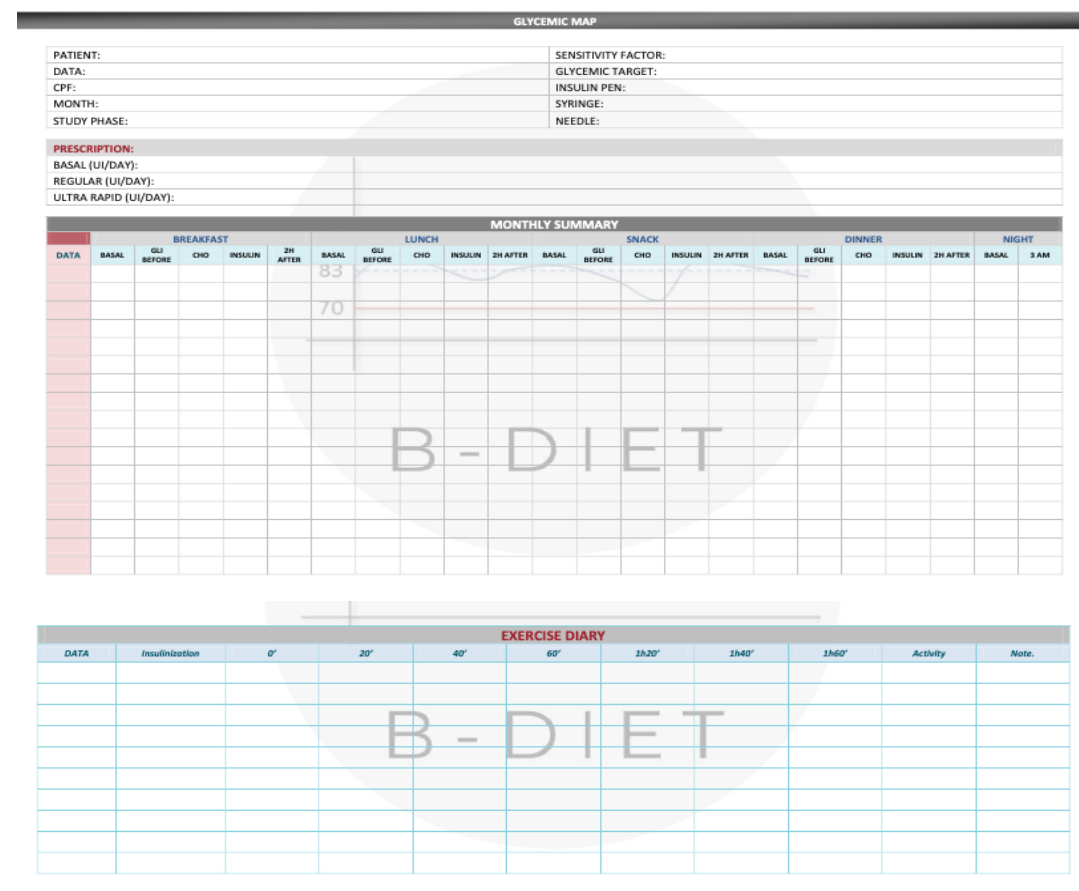

Figure 2: Glycemic map.

\section{Hyperglycemia correction}

Pre-prandial hyperglycemia must be corrected with blood glucose above $140 \mathrm{mg} / \mathrm{dl}$; post-prandial hyperglycemia must be corrected with a glycemia equal to or greater than $180 \mathrm{mg} / \mathrm{dl}, 2$ hours after the meal, with half the dose of insulin.

When pre-prandial blood glucose is equal to or greater than $180 \mathrm{mg} / \mathrm{dl}$, correction should performed according to the following formula.

$$
=\frac{\begin{array}{c}
\text { Correction } \\
\text { Current blood glucose }- \text { target blood glucose } \\
(100 \mathrm{mg} / \mathrm{dl})
\end{array}}{\text { Sensitivity factor }(\text { according to the situation })}
$$

For correction of postprandial hyperglycemia and between hours 22:00 and 06:00 in the morning, the sensitivity factor 100 should be used. In other situations, the sensitivity factor 50 should be adopted. The sensitivity factor will be determined according to the participant's individuality.

\section{Correction of hypoglycemia}

Hypoglycemia occurs when blood sugar level is below 70 $\mathrm{mg} / \mathrm{dl}$; and can be classified as: level 1 - relatively low blood glucose levels: 70 to $55 \mathrm{mg} / \mathrm{dl}$; level 2 - very low blood glucose levels: below $55 \mathrm{mg} / \mathrm{dl}$; and level 3 cognitive impairment requiring help.

For treating hypoglycemia, the hypoglycemia protocol (Figure 3), built specifically for this research, and should be followed. In case of suspected hypoglycemia, the blood glucose must be checked at the fingertip to determine the level of hypoglycemia. For blood glucose between 55 $\mathrm{mg} / \mathrm{dl}$ and $<70 \mathrm{mg} / \mathrm{dl}$, sugar diluted in water should be ingested. The Table 1, showing the impact in blood glucose after oral administration of $1 \mathrm{~g}$ of sugar; according to the participant's body weight $(\mathrm{kg})$ should be used to calculate the amount of sugar to be ingested. The participant must check the blood glucose from the "fingertip" every 15 minutes after ingestion of the sugar diluted in water; until returning to the glycemic goal of, $100 \mathrm{mg} / \mathrm{dl}$. In addition the participant must check glycemia after 1 hour of treatment, when glycemic goal is reached, for safety. If the participant is about to have a meal, she must wait for the blood glucose to be on target, apply the dose of insulin for the meal, wait for time for the insulin action, and, only then, start to eat. For blood glucose levels below $54 \mathrm{mg} / \mathrm{dl}$, or if the participant has severe symptoms, it is recommended to use Gli Instan, which corresponds to $15 \mathrm{~g}$ of glucose. It is not advisable to rely solely on blood glucose sensors correcting for hypoglycaemia. The measurement of capillary blood glucose is essential because, of the sensor delay interstitial blood glucose, which is slower to reflect sudden changes in plasma glucose. The hypoglycemia protocol is depicted in the Figure 3 .

Hypoglycemia must be corrected individually, according to the table given.

\section{Blood glucose management before meals}

Participants will be instructed to correct lower blood glucose levels before the meal as follows: if the blood sugar level is $<70 \mathrm{mg} / \mathrm{dl}$, the participants must correct it 
with sugar in the required amount according to the Table 1. After administering the sugar diluted in water, the volunteers will be instructed to wait 15 minutes and check the capillary blood glucose and check if the blood glucose level has returned to the target $(100 \mathrm{mg} / \mathrm{dl})$. If blood glucose was at the glycemic target $(100 \mathrm{mg} / \mathrm{dl})$, participants will be instructed to administer insulin to cover the meal. If the blood glucose is below $70 \mathrm{mg} / \mathrm{dl}$, the participant must repeat the protocol until the hypoglycemia is corrected.

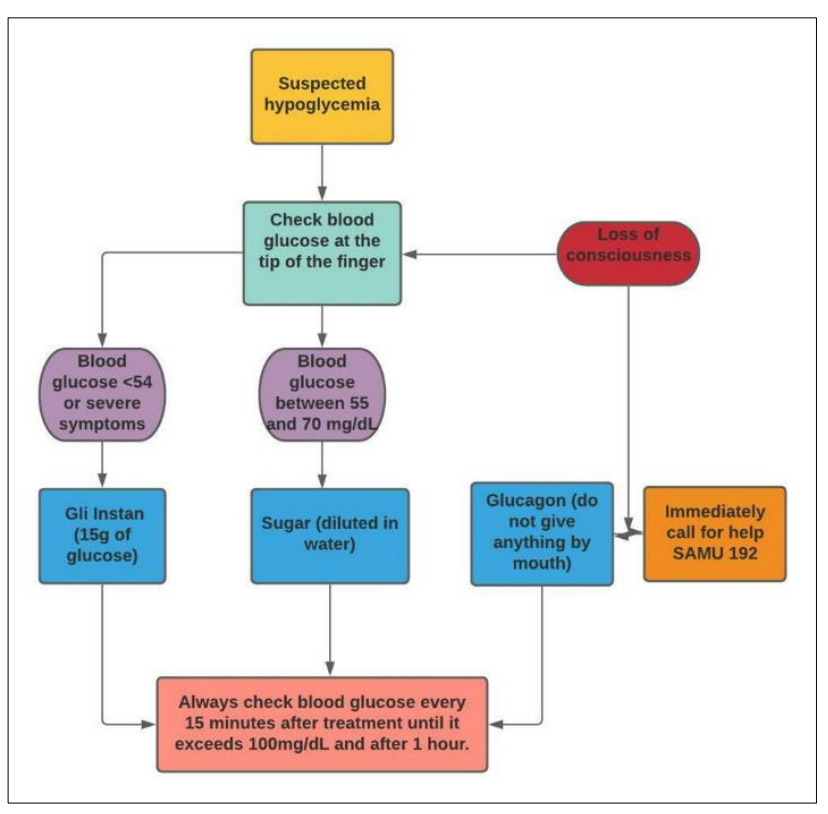

Figure 3: Hypoglycemia correction protocol.

Table 1: Blood glucose elevation with $1 \mathrm{~g}$ of sugar (sucrose) according to body weight (kg).

\begin{tabular}{|l|l|}
\hline Body weight $(\mathrm{kg})$ & $\begin{array}{l}\text { Elevation of blood glucose in } \\
\mathbf{1} \text { g of sugar (glucose) }(\mathbf{m g} / \mathbf{d l})\end{array}$ \\
\hline $\mathbf{1 6}$ & 20 \\
\hline $\mathbf{3 2}$ & 10 \\
\hline $\mathbf{4 8}$ & 7 \\
\hline $\mathbf{6 4}$ & 5 \\
\hline $\mathbf{8 0}$ & 4 \\
\hline $\mathbf{9 5}$ & 3.3 \\
\hline $\mathbf{1 1 1}$ & 3 \\
\hline $\mathbf{1 2 8}$ & 2.5 \\
\hline $\mathbf{1 4 3}$ & 2.2 \\
\hline
\end{tabular}

\section{Diet adaptation symptoms - "low carb flu"}

Participants will be informed about possible symptoms of adaptation to the diet, caused by the reduction of plasma insulin and an initial dehydration, with urinary losses of some electrolytes, such as magnesium and potassium.

They will also be instructed to eat foods with more magnesium, potassium and to increase their salt intake.
During the study, all participants will receive $400 \mathrm{mg}$ of chelated magnesium at bedtime.

\section{Insulins}

Type 1 diabetic participant routinely use basal insulin to suppress hepatic neoglycogenesis and maintain controlled fasting blood glucose over 24 hours. The basal insulins used were chosen according to availability and participant preference, but were restricted to the following insulins: Degludeca, Detemir and Glargina. Basal insulin was divided into 2 doses to better cover 24 hours. In addition to basal insulin, the participant will use fast and ultra-fast insulins to cover the macronutrients in meals (carbohydrates, proteins and fats). To cover carbohydrates, participants will use rapid insulin Lispro (Humalog), Asparte (Novorapid), Glulisine (Apidra) or Asparte (Fiasp). For proteins and fats, the participant will use regular insulin. Rotation of insulin application sites will be advised to prevent lipodystrophy, which is characterized by a deformity in the subcutaneous tissue, resulting in impaired insulin absorption, causing a risk of hyperglycemia and promoting greater variability in blood glucose control. Insulin usage will be recommendations. The syringe for insulin BD ultra-fine $6 \mathrm{~mm}$ graduated $1 / 2$ in $1 / 2$ units was standardized for insulin application. Insulin pens will only be used for the application of larger doses, as is the case of basal insulin.

\section{Time for insulin application before meals}

Participants will be instructed to wait 15 minutes after insulin application before having a meal. However, the application time will be evaluated and adjusted according to the individual needs of each participant.

\section{Food bolus}

To cover the carbohydrates, participants will be instructed to consider that $100 \%$ of them are converted into glucose. Thus, the total carbohydrates consumed in the meal would be divided by 15 , taking into account the initial ratio of $1: 15$, i.e. 1 unit of fast insulin covering $15 \mathrm{~g}$ of carbohydrates. It is important to remember that the insulin to carbohydrate ratio will be determined individually.

To cover the proteins, participants will be instructed to consider that $50 \%$ of them would be transformed into glucose. Therefore, participants will calculate the insulin dose with the same ratio used in carbohydrate counting (1:15); but will use regular insulin to cover proteins instead.

Participants will be instructed to consider fat in the count only when ingesting more than $30 \mathrm{~g}$ of fat in the meal. Fat is a macronutrient with little impact on blood glucose, if ingested in small amounts; however, above $30 \mathrm{~g}$ per meal, it fat usually starts to have a greater and later impact on blood glucose. In this study, $30 \%$ of the fat in the meal is expected to be transformed into glucose. Hence, 
participants will calculate the insulin dose with the same ratio used in the carbohydrate count (1:15); but will use regular insulin to cover fats instead.

If blood glucose is high before the meal, correction should be performed; as explained in the above flowchart. The calculated dose of rapid insulin for correction should be added to the prandial bolus to cover the carbohydrates and applied together. Regular insulin to cover protein and fat should also be added and applied at the same time.

\section{Construction and standardization of $130 \mathrm{~g}, 90 \mathrm{~g}, 50 \mathrm{~g}$ and 30 g carbohydrate diets}

To design the diets, foods were standardized into groups according to the source of macronutrients carbohydrates, such as, fruits, cereals, pulses, grain or cereal flour, leafy, low-starch vegetables, and fruit pulp; proteins, such as, fatty meats/fish, lean meats/fish, eggs, fatty cheeses, milk and yoghurt, low-fat cheese, and cottage cheese; and fats, such as, oilseeds, seeds, cashew nuts, and avocado. The participants must complete the diet sheet every day, according to the foods chosen within each proposed food group. Table 2 lists, the amount of macronutrients in each food group.

Table 2: Food and macronutrient group.

\begin{tabular}{|ll|}
\hline Coods & Amount (g) \\
\hline Cereal & 12 \\
\hline Legumes & 12 \\
\hline Grain or cereal flour & 4 \\
\hline Tubercules & 12 \\
\hline Sweet fruits & 4 \\
\hline Leafy vegetables & 2 \\
\hline Low starch vegetable & 4 \\
\hline Low carb fruits & 4 \\
\hline Fruit pulp & 4 \\
\hline Proteins & \\
\hline Egg white & 3 \\
\hline Fat meats/fish & 25 \\
\hline Lean meats/fish & 25 \\
\hline Eggs & 6 \\
\hline Fat cheeses & 4 \\
\hline Milk and yoghurts & 3 \\
\hline Slim cheeses & 4 \\
\hline Cottage cheeses & 4.7 \\
\hline Fats & 5 \\
\hline Fats & 10 \\
\hline Oleaginous & 5 \\
\hline Seeds & 6.4 \\
\hline Cashew nut & 5.8 \\
\hline Avocado & \\
\hline
\end{tabular}

Using the desire amount of macronutrient in each food group, the portion size was created, allowing participants to arrive at equivalences, and to promote substitutions within each food group. Food composition table of this protocol was created based on tables such as Taco, Tucunduva or USDA were used to arrive at equivalence and allow for substitutions within the proposed food groups. Macronutrients quantities were kept fixed across meals to make blood glucose management simple. Ideally, standardized macronutrients should be used to calculate serving size.

For example, knowing that $100 \mathrm{~g}$ of baroa potato contains $18.9 \mathrm{~g}$ of carbohydrates, a portion of $12 \mathrm{~g}$ carbohydrates would have $63 \mathrm{~g}$ of baroa potato, the following calculation should be used.

To find the amount of 1 portion of the tuber. Consider that the serving has $12 \mathrm{~g}$ of carbohydrates.

$100 \mathrm{~g}$ baroa potato $=18.9 \mathrm{~g}$ carbohydrates

$X=12$ g carbohydrates

$X=63$ g baroa potato

To find amount of lipids for a $63 \mathrm{~g}$ of baroa potato (12 $\mathrm{g}$ of carbohydrates), the following calculation should be used.

$100 \mathrm{~g}$ baroa potato $=0.2 \mathrm{~g}$ of lipids

$63 \mathrm{~g}$ baroa potato $=X$

$X=0.12 \mathrm{~g}$ of lipids

To find the amount of protein present in $63 \mathrm{~g}$ of baroa potato (12 $\mathrm{g}$ of carbohydrates), the following calculation should be used.

$100 \mathrm{~g}$ of baroa potato $=0.9 \mathrm{~g}$ of protein

$63 \mathrm{~g}$ of baroa potato $=X$

$X=0.5 \mathrm{~g}$ of protein

The portion size of each food group was determined based on the amount of carbohydrates in each diet. The diet was constructed from the amount of carbohydrates consumed daily, distributed in four daily meals (breakfast, lunch, snack and dinner), considering the food needs of each group of individuals distributed by sex and height. The diet started with $130 \mathrm{~g}$, then $90 \mathrm{~g}, 50 \mathrm{~g}$ and finally, $30 \mathrm{~g}$ of carbohydrates, if required. The objective was to gradually decrease the amount of carbohydrates and reduce the glycemic load and glycemic index of foods consumed by participants.

\section{DISCUSSION}

Despite advances in treatments for type 1 diabetes mellitus, glycemic control still remains unsatisfactory. After the discovery of insulin, the recommendation 
became "eat what you want and apply insulin", which is ineffective in achieving normal glycated hemoglobin and reducing glycemic variability. High levels of glucose in the bloodstream are harmful and can lead to various shortand long-term complications in the body, such as cardiovascular problems, stroke, hypertension, limb amputation, among others. After the discovery of insulin, guidelines recommended $50 \%$ to $60 \%$ of calories coming from carbohydrate consumption for individuals with diabetes.

However, this recommendation is ineffective, since carbohydrate is the macronutrient that causes the greatest increase in blood glucose, directly impacting postprandial blood glucose, the main determinant of Hb1Ac. The current method, which is based on determining insulin doses according to the macronutrients of the meal, by counting carbohydrates, is inaccurate, unpredictable and does not eliminate errors, resulting in greater risks to the participants.

In contrast, the low-carbohydrate diet is an effective and safe way to control diabetes; and its effectiveness has been recognized since 2019, albeit timidly by the ADA, for the treatment of type 2 diabetes. Given this context, an intervention study, in which each participants is her own control, is extremely important, to investigate and provide the scientific community with concrete and accurate data on the approach of the low-carbohydrate diet in the treatment of type 1 diabetes.

It is important to seek treatments that are promising, to provide a better quality of life for individuals with type 1 diabetes, to reduce complications arising from inadequate control, and to provide guidelines for effective and concrete information for the management of type 1 diabetes. From a socioeconomic point of view, it is much better to invest in diabetes education and prevention than to treat the direct complications of the disease (amputations, hemodialysis and blindness) or indirect ones (diabetes as a complicating factor for any other acute or chronic comorbidity).

\section{CONCLUSION}

For type 2 diabetes, the effectiveness of a lowcarbohydrate diet for treating the disease is already clear. However, for type 1 diabetes, the guidelines still do not recommend the use of a low-carbohydrate diet. Therefore, there is a need for effective studies that show that the lowcarbohydrate diet is a safe strategy, which reduces medications, reduces hypoglycemia and hyperglycemia, improves the quality of food, and thus provides better quality of life and health the patient, and in the long term, reduces the complications of this condition

\section{ACKNOWLEDGEMENTS}

Authors would like to thank The Clinic, in Belo Horizonte, Minas Gerais, for the partnership for the development of the proposed research. The Federal University of Minas Gerais for supporting the development of the study. The CAPES (Coordination for the Improvement of Higher Education Personnel). In short, all professionals involved in the research.

\section{Funding: No funding sources}

Conflict of interest: None declared

Ethical approval: The study was approved by the University of Minas Gerais human research ethics committee, under the number 4.961 .640

\section{REFERENCES}

1. ADA. American Diabetes Association. Diagnostics and classification of diabetes Mellitus. Diabetes Care. 2014;37:81-90.

2. Cobo-Vuilleumier N, Gauthier BR. Time for a paradigm shift in treating type 1 diabetes Mellitus: coupling inflammation to islet regeneration. Metabolism Clinical and Experimental. 2020;104(154137).

3. DiMeglio L, Evans-Molina C, Oram RA. Type 1 diabetes. Lancet. 2018;391(10138):2449-62.

4. International Diabetes Federation. Atlas de Diabetes da IDF, $9^{\circ}$ edição, 2019. Available at: https://www.diabetesatlas.org/en/. Accessed on 25 August 2021.

5. World Health Organization. Diabetes. 2020. Available at: https://www.who.int/news-room/fact sheets/detail/diabetes\#: :text=Diabetes $\% 20$ is $\% 20 \mathrm{a}$ $\% 20$ major\%20causehigh\%20blood\%20glucose $\% 20 \mathrm{i}$ n\%202012. Accessed on 14 September 2021.

6. Brazilian diabetes society. Brazilian Society of Diabetes Guidelines 2019-2020, 2019.

7. ADA. American Diabetes Association. Statistics about Diabetes. 2018. Available at: https://www.diabetes.org/resources/statistics/statisti cs-about-diabetes. Accessed on 20 May 2020.

8. Kahanovitz I, Sluss PM, Russell SJ. Type 1 diabetes - A clinical perspective. Point Care. 2018;16(1):3740.

9. Casqueiro J, Casqueiro J, Alves C. Infections in patients with diabetes Mellitus: A review of pathogenesis. Indian $\mathrm{J}$ Endocrinol Metab. 2012;16(1):27-36.

10. Atkilt HS, Turago MG, Tegegne BS. Clinical Characteristics of Diabetic Ketoacidosis in Children with Newly Diagnosed Type 1 Diabetes in Addis Ababa, Ethiopia: A Cross-Sectional Study. PLoS One. 2017;12(1):e0169666.

11. Benoit SR; Zhang Y, Geiss LS, Gregg EW, Albright A. Trends in Diabetic Ketoacidosis Hospitalizations and In-Hospital Mortality - United States, 20002014. Centers for Disease Control and Prevention. 2018;67(12):362-5.

12. Duca LM, Wang B, Rewers M, Rewers A. Diabetic Ketoacidosis at Diagnosis of Type 1 Diabetes Predicts Poor Long-term Glycemic Control. Diabetes Care. 2017;40(9):1249-55. 
13. Dabelea D, Rewers A, Stafford JM, Standford DA, Lawrence JM, Saydah S, Imperatore G, Mayer-Davis E J, Pihoker C. Trends in the prevalence of ketoacidosis at diabetes diagnosis: the SEARCH for diabetes in youth study. Pediatrics. 2014;130(4):93845.

14. Turton JL, Raab R, Rooney KB. Low-carbohydrate diets for type 1 diabetes Mellitus: A systematic review. Plos One. 2018;13(3):e0194987.

15. Feinman RD, Pogozelski WK, Astrup A, Bernstein RK, Fine EJ, Westman E, et al. Dietary carbohydrate restriction as the first approach in diabetes management: Critical review and evidence base. Nutrition. 2015;31(1):1-13.

16. Moreira ED, Neves RCS, Nunes ZO, Almeida MCCA, Mendes ABV, Fittipaldi JASF, Ablan F. Glycemic control and its correlates in patients with diabetes in Venezuela: Results from a nationwide survey. Diabetes Research and Clinical Practice. 2010;87(3):407-14.

17. Sastre J, Pinés P J, Moreno J, Aguirre M, Blanco B, Calderón D, Herranz S, Roa C, Lopez J. Metabolic control and treatment patterns in patients with type 1 diabetes in Castilla-La Mancha: the Diabetes type 1 in Castilla La Mancha study. Endocrinología y Nutrición. 2012;59(9):539-46.

18. De Souza ACCB, Felício JS, Koury CC, Neto JFAN, Miléo KB, Santos FM, et al. Health-related quality of life in people with type 1 Diabetes Mellitus: data from the Brazilian Type 1 Diabetes Study Group. Health and Quality of Life Outcomes. 2015;13(1):204.

19. Craig M, Twigg S, Donaghue K. National evidencebased clinical care guidelines for type 1 diabetes in children, adolescents and adults. 2011. Available at: https://www.google.com/url?sa=t\&rct=j\&q=\&esrc= $\mathrm{s} \&$ source $=$ web $\& \mathrm{~cd}=\& \mathrm{cad}=\mathrm{rja} \&$ uact $=8 \& \mathrm{ved}=2 \mathrm{ahU}$ KEwi27aWf5rj1AhUGzjgGHWQBD-

4QFnoECAgQAQ\&url=https $\% 3 \mathrm{~A} \% 2 \mathrm{~F} \% 2 \mathrm{Fdiabetes}$ society.com.au\%2Fdocuments\%2FType1guidelines 14Nov2011.pdf\&usg=AOvVaw1Bc6Vk43pzDAdn9 jhJjEPw. Accessed on 20 May 2020.

20. McKnight JA, Wild SH, Lamb MJ. Glycaemic control of Type 1 diabetes in clinical practice early in the 21st century: an international comparison. Diabetic Med J Bri Diabetic Assoc. 2015;32:103650.

21. Juutilainen A, Lehto S, Rõnnemaa T, Pyorala K, Laakso M. Similarity of the impact of type 1 and type 2 diabetes on cardiovascular mortality in middleaged subjects. Diabetes Care. 2008;31(4):714-9.

22. Kelly CT, Mansoor J, Dohm GL. Hyperinsulinemic syndrome: the metabolic syndrome is broader than you think. Surgery. 2014;156:405-11.

23. Wang MY, Yu X, Lee Y. Latrogenic hyperinsulinemia in type 1 diabetes: its effect on atherogenic risk markers. Journal diabetes its complications. J Diabetes Complications. 2013;27:70-4.
24. Kolic J, Johson JD. Promises and pitfalls of beta cellreplacement therapies. Nature Metabolism. 2021;3:1036-7.

25. Wang MY, Yu X, Lee Y, McCorkle K, Clark GO, Strowig S, Unger RH, Raskin P. Iatrogenic hiperinsulinemia in type 1 diabetes: Its effect on atherogenic risk markers. J Diabetes Complic. 2013;27(1):70-4.

26. Bahia L, Kupfer R, Momesso D, Cabral DAP, Tschiedel B, Puñales M, et al. Health-related quality of life and utility values associated to hypoglycemia in patients with type 1 diabetes Mellitus treated in the Brazilian Public Health System: a multicenter study. Diabetology \& Metabolic Syndrome. 2017;9:9.

27. Orozco-Beltrán D, Artola S, Jansá M, Torre-Casares ML, Fuster E. Impact of hypoglycemic episodes on health-related quality of life of type- 2 diabetes Mellitus patients: development and validation of a specific QoLHYPO@ questionnaire. Health and Quality of Life Outcomes. 2018;16(52).

28. Bassuk S S, Manson J E. Epidemiological evidence for the role of physical activity in reducing risk of type 2 diabetes and cardiovascular disease. J Appl Physiol. 2005;99(3):1193-204.

29. Sami W, Ansari T, Butt NS, Hamid MRA. Effect of diet on type 2 diabetes Mellitus: A Review. Int J Health Sci (Qassim). 2017;11(2):65-71.

30. Sainsbury E, Kizirian NV, Partridge SR. Effect of dietary carbohydrate restriction on glycemic control in adults with diabetes: A systematic review and meta-analysis. Diabetes Rese Clin Practice. 2018;139:239-52.

31. Patton SR. Adherence to Diet in Youth with Type 1 Diabetes. J Am Diet Assoc. 2011;111(4):550-5.

32. Oosterveer P. Globalization and sustainable consumption of shrimp: Consumers and governance in the global space of flows. Int $\mathbf{J}$ Consumer Stud. 2006;30(5):465-76.

33. Rahati S, Shahraki M, Arjomand G, Shahraki T. Food Pattern, Lifestyle and Diabetes Mellitus. International Journal of High Risk Behaviors and Addiction. 2014;3(1):e8725.

34. Wylie-Rosett J, Aebersold K, Conlon B, Isasi CR, Ostrovsky NW. Health effects of low-carbohydrate diets: Where should new research go? Current Diabetes Reports. 2013;13(2):271-8.

35. Ludwig DS, Ebbeling CB. The carbohydrate-insulin model of obesity. JAMA Internal Medicine. 2018;178(8):1098.

36. Wolpert HA, Atakov-Castillo A, Smith SA, Steil GM. Dietary fat acutely increases glucose concentrations and insulin requirements in patients with type 1 diabetes: implications for carbohydratebased bolus dose calculation and intensive diabetes management. Diabetes Care. 2013;36:810-6.

37. Osler $\mathrm{W}, \mathrm{McCrae} \mathrm{T}$. The principles and practice of medicine: New York, London, D. Appleton and company. 1920;1201.

38. Nielsen JV, Gando C, Paulsson JC. Low carbohydrate diet in type 1 diabetes long-term 
improvement and adherence: A clinical audit. Diabetology \& Metabolic Syndrome. 2012;4(1):23.

39. Krebs JD, Strong AP, Cresswell P, Reynolds AN, Hanna A, Haeusler S. A randomised trial of the feasibility of a low carbohydrate diet vs standard carbohydrate counting in adults with type 1 diabetes taking body weight into account. Asia Pacific Journal of Clinical Nutrition. 2016;25(1):78-84.

40. Buyken A E, Toeller M, Heitkamp G. Carbohydrate sources and glycaemic control in Type 1 diabetes
Mellitus. EURODIAB IDDM Complications Study Group. Diabetic medicine J Bri Diabetic Association. 2000;17:351-9.

Cite this article as: Felizardo AA, Paiva CDSB, Brasileiro JDFL, Souza MSDD, Correa RM, Carvalho DRD, et al. Implementing a low-carbohydrate diet in adults to manage type 1 diabetes mellitus: a standard of care protocol. Int J Clin Trials 2022;9(1):18-28. 